\title{
A Real-Time Directed Routing Protocol Based on Projection of Convex Holes on Underwater Acoustic Networks
}

\author{
Hu HongNing, Liu Zhong and Li Lu \\ Electronics Engineering College, Navy University of Engineering, Wuhan, Hubei Province, china
}

\begin{abstract}
The directed routing protocol ends in failure when it faces a situation of the destination node with a very low velocity in a sparse ad hoc network so that none of nodes exist in its forwarding zone. Illuminated by BFDREAM and ZONER, the paper firstly proposes a novelty routing protocol that is fairly immune to forwarding failure through projecting the present source node on the boundaries of convex baffle holes of underwater acoustic networks in deep sea. Compared with DREAM and BFDREAM, the experimental results show our protocol achieves a great improvement in decreasing the propagation delay and reducing quantities of the non-effect information. So the new protocol may have a bright application prospect in deep sea acoustic networks.
\end{abstract}

Index Terms: Directed flooding; sparse; non-effect; acoustic networks in deep sea; convex baffle holes

(C) 2012 Published by MECS Publisher. Selection and/or peer review under responsibility of the Research Association of Modern Education and Computer Science

\section{Introduction}

The main idea of directed routing protocols is to reduce the possibility that the broadcast storm [1] appears by limiting the forwarding range of packets partly. This kind of protocols does not need to maintain a routing table and has good robustness, so it is praised highly by most routing protocols designed for underwater acoustic networks [2].

\section{Related works}

DREAM mentioned in [3] specifies that the each forwarding node including the source node computers an angle range called the destination node's direction firstly, and then forwards packets to all neighbor nodes in the destination direction. Reference [4 5] offers a new thought to reduce the cost and energy consumption of networks by replacing broadcasting of traditional flooding protocols with forwarding packets only in a virtual forwarding range. Reference [5] steps forward in calculating the virtual forwarding by using a message of

* Corresponding author.

E-mail address: huhongning@ hotmail.com, synorsmith@ hotmail.com 
acknowledges pruning the forwarding range. No matter what methods are used for improving their performance, the essence of directed protocols is based on flooding protocols. So this kind of protocols is relatively suitable for the networks that expect high dependability but not frequent of data transmission and easy to fail in a sparse network with the destination node moving at a very low speed [6]. For convenience explain, we call this situation as the hole phenomenon.

Reference [6-7] offer a similar idea to improve this situation by traversing nodes on boundaries of the hole, and are utterly different from each other in implement mechanism and application circumstance. The BFDREAM protocol proposed in [6] is designed for underwater acoustic networks, which requires every packet be forwarded within an included angle scope of one-hop-nodes and only those one-hop-nodes who have never received the packet before deal with it. When a packet is blocked in a hole zone, BFDREAM continues the transmission through choosing the node from all one-hop-neighbors who has the minimum angle with the destination direction as the next forwarding node. And the ZONER protocol [7] is designed for Mobile Sensor Networks, which needs the information about the hole boundaries in advance to bypass the zone from clockwise and counterclockwise direction respectively. Compared with ZONER, BFDREAM is easy for implement and requires a small amount of computation and does not require nodes maintaining a neighbor table during transmission. But this protocol also has some shortcomings, for example, it cannot guarantee the success of a packet delivery in spite of there exists a way in the network to the destination node.

For the network topology shown in Fig. 1, BFDREAM falls into failure and DREAM finds ways to send packet to the destination successfully (Fig. 2).

Taken the disadvantages of BFDREAM into account, the paper proposes a novelty routing protocol aimed at the convex baffle holes in underwater acoustic networks. Our protocol can guarantee the success of packets delivery through that it finds out the projection node and the present destination node and the guide node from the boundaries of convex holes in network, which can bypass the convex holes with little cost and delivery packets back to the directed forwarding zone. The remainder of this paper is organized as follows: In section III, we present some related definitions and theorems firstly, and then describe our new protocol in detail; the simulation results and analysis are presented in section IV and finally section V concludes the paper and points out the future research direction.

\section{A new protocol based on projection of convex holes}

\section{A. The fundamental principle of the projection}

Given $S$ and $D$ as the source node and the destination node of packet respectively and none of them are isolated points of network; we define the forwarding zone $Z$ according to the movement factors of the destination node. As shown in Fig. 3, the hole $H$ obstructs $Z$.

So the fundamental principle of the projection goes as follows:

1) Let assume $S D$ as the line segment connected with $S$ and $D$, and then $S D$ separates $H$ into two parts from which the protocol will find out the boundary node who has the maximum anger with $S D$ respectively. Here we call the two nodes as $S_{u p}^{\prime}$ and $S_{d o w n}^{\prime}$, and then pick up the node with less anger from the nodes as the guide node $S_{d}$. Finally, the protocol will respectively find out a node from two continuous edges of $H$ left in $Z$ who has the shortest distance to $S_{d}$, and chooses the one from the two nodes who has the longer distance to $S$ as the projection node $S^{\prime}$ and the other as the present destination node $D^{\prime}$.

2) The protocol requires packets delivery from $S$ to $D$ in the way of directed routing, and then forwards packets from $D^{\prime}$ to $S^{\prime}$ along the boundaries of $H$ under the guidance of $S_{d}$. When $S^{\prime}$ receives packets, the protocol sets $S$ as the present node and $H$ has been bypassed.

Before describing the new protocol, we will present some related definitions and theorems in the following subsection. 


\section{B. Related Definitions and Theorems}

The Let assume the topology of network be $G$.

Definition 1. The hole $H$ consists of the void-area $V$ and the area-boundary $B$; A hole $H$ is a convex hole if

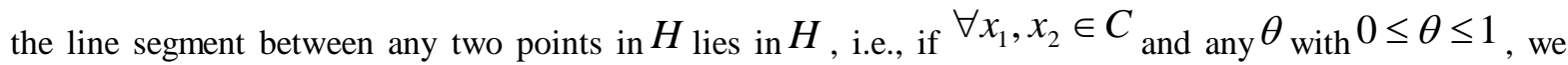
have $\theta x_{1}+(1-\theta) x_{2} \in C$. For simplicity, we call the hole as the convex hole in the remainder of paper.

Definition 2. Given $B=(K, L)$ as the area-boundary of $H$, among which $K=\left\{k_{1}, k_{2}, \cdots, k_{n}\right\}$ is the set of boundary nodes and $L=\left\{l_{1}, l_{2}, \cdots, l_{n}\right\}$ is the set of the connected relationship among nodes in $K$; we define $B_{r}$ as one of continuous edges of $B$, if ${ }^{B}$ satisfies the following conditions:

1) $B_{r}=\left(K_{r}, L_{r}\right) \subseteq B$;

2) $K_{r}=\left\{k_{r}, k_{r+1}, \cdots, k_{r+m}\right\} \subseteq K, 1 \leq r, 0 \leq m, r+m \leq n \quad L_{r}=\left\{l_{r}, l_{r+1}, \cdots, l_{r+m}\right\} \subseteq L$;

Definition 3. There exist a connected region $Z$ and a hole $H$ in network $G$. If all boundary nodes of $H$ are removed from $G, A$ becomes two separate parts $a_{1}, a_{2}$; here we define this situation as $H$ obstructs $Z$.

Definition 4. Given a hole $H$ and a node $A$, if $H \subseteq G$ and $A \in G$, the distance between $A$ and $H$ is:

$$
D_{A H}=\left\{\begin{array}{cl}
\underset{i=1,2, \cdots, n}{\min \left\{D_{A k_{i}}\right\}} & , A \notin H \\
0 & , A \in H
\end{array}\right.
$$

In which $K$ is the boundary set of $H$ and $K=\left\{k_{1}, k_{2}, \cdots, k_{n}\right\}$.

Theorem 1 . The judgment whether $H$ obstructs $Z$ or not.

Assume $H$ as the convex hole and $Z$ as the forwarding zone of packets. If $H$ satisfies any one following condition, $H$ does obstruct $Z$.

1) Given $S$ and $D$ as the source node and the destination node of packet respectively and the statement of $S \notin H \cap D \notin H$ is false.

2) Assume $B_{r 1}, B_{r 2} \subset B$ and $B_{r 1} \neq B_{r 2}$, and the statement of $B_{r 1}, B_{r 2} \subset Z$ is true. Here, $B_{r 1}$ and $B_{r 2}$ may be a segment of $l_{i}$ and $l_{j}$ respectively, and $i \neq j$, as shown in Fig. 4.

Proof.

1) According to definition 3, the statement is clearly established. There lies no way from the source to the destination.

By contradiction, Suppose $H$ does not block the forwarding domain $Z$.

2) Let $H$ go into $Z$ in two continuous edges were $B_{x}, B_{y} \subseteq B$, so $B_{x}$ and $B_{y}$ has no common nodes and common edges according to Definition 2. Set $B_{x}$ and the boundaries of $Z$ intersect at $z_{x_{1}}, z_{x_{2}}$, and $B_{y}$ intersect with $Z$ at $z_{y_{1}}, z_{y_{2}}$; those four points must be different from each other, and can not be in the same line according to Definition 1; further, any three of the four points are not collinear. Given the curve segment $z_{x_{1}} z_{y_{2}}$ is part of one of $Z$ 's boundaries and the curve segment $z_{x_{1}} z_{y_{2}}$ is part of another, $z_{x_{1}} z_{y_{2}}$ and $z_{x_{1}} z_{y_{2}}$ and $B_{x}$ and $B_{y}$ consist of a sub-region $h \subset H$, also $h \subset Z$; so $Z$ will become two separate parts $a_{1}$ and $a_{2}$ after removing $h$ from $Z$. This conflicts with Definition 3 . 


\section{The New Protocol Described in Details}

Our protocol consists of two parts, which are the hole boundary detection part and the packet transmission part respectively.

1) The hole boundary detection part.

This part is carried out under the network idle, which adopts the protocol in [8] and accomplishes the boundaries detection of all holes in network before the packet transmission part begins. For example, this part figures out the hole boundary of the network shown in Fig. 5 with the red points.

2) The packet transmission part.

This part is the core of our protocol, and it goes as follows: as shown in Fig. 6

a) The protocol sets the source node of packet as the present node $S$, and then goes into step (b).

b) According to $S$ and the destination node $D$ and the movement factors of $D$, the protocol determines the forwarding zone $Z$ and then goes into step (c).

c) Based on Theorem 1, the protocol will pick out the set of the convex baffle hole $W=\{H\}$; if there do not exist a hole obstructing $Z$,e. g., $W=\phi$, the protocol will go into step (g); otherwise it goes into step (d).

d) Find out the hole $H=(B, V)$ who has the shortest distance to $S$ and determine whether the statement of $D_{S H}>0$ is true or not; if the statement is true, the protocol goes into step (e); Otherwise the protocol terminates and broadcasts an err message.

$e$ ) Find out the present destination node $D^{\prime}$ and the guide node $S_{d}$ and the projection node $S^{\prime}$ from the set of $B$ according to the fundamental principle presented in section A; and then goes into step (f).

f) Transmit the packet to node $D^{\prime}$ with the directed flooding method in the forwarding zone, and then delivery the packet form $D^{\prime}$ to $S^{\prime}$ as the guidance of $S_{d}$; Finally, the protocol goes into step (g).

g) Set $S^{\prime}$ as the present node $S$, the protocol goes into step (b).

h) Transmit the packet to the destination node $D$ with the directed flooding method, and the network goes back the idle state.

Theorem 2. Whether there are some holes in the network or not, our protocol shall accomplish the transmission of packets successfully if there lay one way from the source node to the destination node at least.

Proof. During the transmission of packets, our protocol will maintain the transmission mode of the directed routing till it meets one convex baffle hole in its forwarding zone. If there does not exist such a hole in the network, the statement is clearly establishment. Otherwise, one of packet delivery routes from the source node to the destination node shall be made up of some sub-routes which can be classified four kinds: the first one is the route from the source node to the boundary of hole; the second one is a part of the boundary; the third one is the route from one hole boundary to another; the last one is the route from the last boundary to the destination node. Obviously, this route is just coincided with the one constructed by our protocol.

At the end of this section, we present the actual packet delivery route with the new protocol in the network of Fig. 1, as shown in Fig. 7.

\section{Simulation results}

The paper will use DREAM and BFDREAM as references to check the performance of the new protocol. There are two kinds of node in the network, which are the super node and the normal node respectively. The former takes charge of data collection and packet creation and the latter is only responsible for packet forwarding. 
The former has the ability of voluntary movement, and the latter can only follow the deep sea flow making random drift. Experimental environment and related parameters are introduced in the following paragraphs.

In this experiment, 500 normal nodes were arranged in a square area of $20 \times 20$ square kilometers, while two super nodes kept moving at a constant speed with random variation in direction. The longest communication distance of every node is 1000 meters; the speed of super nodes is 4.12 meters per second, and the acoustic speed is 1500 meters per second, and the rate of data transmission is 9375 bits per second [6]; the size of the packet is 368 bits, and the size of the ACK frame is 20 bits.

The experiment includes two parts; the first part analyzes the process of a certain packet transmission, and the second part tests the protocol's performance compared with that of DREAM and BFDREAM with the situation that two super nodes randomly send 1000 packets to each other within an hour separately.

1) The first part of experiment:

Fig. 8 10 present three different cases about a packet transmission in the network with adopting the three protocols respectively. Obviously, our protocol inherits the robustness of DREAM as well as accomplishes the packet transmission successfully with only a few nodes taking part in transmission.

2) The second part of experiment:

According to [6], mean velocity of ocean current is $1.2 \times 10^{-2} \mathrm{~m} / \mathrm{s}$ whose movement direction is timevarying, so the topological structure of network can approximately be viewed as an unchanged one in one hour and the hole detection part of our protocol does executed only one times before the beginning of experiment. In the following experiment, we will test those protocols' performance from three aspects, such as the average delay, the network energy consumption, and the ratio between the non-effect information and the effect information.

Fig. 11 gives the average delay comparison of three protocols. Clearly, the DREAM protocol has the most serious delay problem among three protocols, and the BFDREAM lays the second place. The phenomenon is caused by the sparse network and the low velocity of the destination node which directly results in there are no nodes involved in the forwarding zone of DREAM, so DREAM needs to keep on invoking the recovery mechanism for packets retransmission; the solution offered by BFDREAM is to bypass all holes immediately, but BFDREAM does not care about the length of transmission route; our protocol wraparounds the forwarding zone along with the shortest boundaries of hole to solve this problem, so it has the least value among three protocols.

Fig. 12 gives the consumption of Network-Energy after finishing all communication tasks. Fig. 13 shows the ratio between the total load of non-effect information and the total load of effect information which have been created during the whole experiment. As shown in Fig.12 and Fig.13, our protocol gains an advantage over BFDREAM and DREAM in reducing the non-effect information and keeping low energy consumption.

\section{Conclusion and future works}

3) According to the challenges faced by underwater acoustic networks, the paper points out the superiority of directed routing protocols among all other routing protocols, and then chiefly studies a typical deep sea acoustic routing protocol called BFDREAM.

4) Inspired by the idea of hole detection of ZONER, we propose a novelty acoustic routing protocol which solves problems faced by BFDREAM through projecting of all convex holes in networks.

5) Simulation results show our new protocol is superior to BFDREAM in decreasing the average delay and the ratio of non-effect information except for inheriting its advantage of low energy consumption and simply operation. And our protocol can stand guarantee for the success of packets transmission along with a relatively short route as well as DREAM does.

6) The future researches will put emphasis on resolving the problems when the baffle hole is non-convex. 


\section{References}

[1] Y.-C. T seng, S.-Y. Ni, and Y.-S. Chen, "The broadcast storm problem in a mobile ad hoc network, "Wireless Networks, vol.8, Issue:2/3, pp.153-167. Kluwer Academic Publisher, May 2002.

[2] I. F. Akyildiz, D. Pompili, and T. Melodia. Underwater Acoustic Sensor Networks: Research Challenges[J]. Ad Hoc Networks, 2005, 3(3): 257-279.

[3] S. Basagni, I. Chlamtac, and V. R. Syrotiuk, "A distance routing effect algorithm for mobility (DREAM), ’Proc. Of MOBICOM’98, Dallas Texas, USA, pp. 76-84, 1998.

[4] R.Farivar, M. Fazeli, and S. G. Miremadi, "Directed Flooding: A fault tolerant routing protocol for wireless sensor networks, ”2005 Systems Communications ( ICW'05, ICHSN'05, ICMCS'05, SENET’05), pp. 395-399, 2005.

[5] J. L. Gao, Y. L. Xu, and X. W. Li, "Directed Flooding with Self-Pruning in Wireless Sensor Networks"Proceedings of the 2007 IEEE International Conference on Integration Technology, Shenzhen, China, March, 2007.

[6] H. N. Hu, Z. Liu, B. Yang, "BFDREAM: A new routing protocol for deep sea acoustic network.'Proceedings of 2010 IEEE 10th international conference on signal processing (ISCP'10), IEEE Press, Oct. 2010, pp.2377-2381.

[7] X. Li, N. Santoro, "ZONER: A ZONE-based Sensor Relocation protocol for Mobile Sensor Networks." In Proc. of IEEE LCN/WLN, pp. 923-930, 2006

[8] Y. Wang, J. Gao, and Joseph S.B. Mitchell. Boundary recognition in sensor networks by topological methods. In MobiCom'06, Los Angeles, California, USA, September 23-26. 2006.

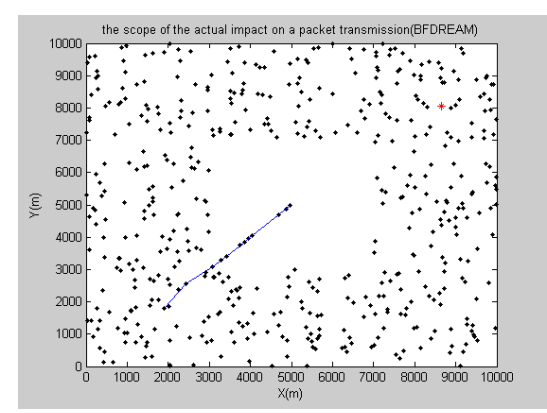

Figure 1. BFDREAM falls into failure in such networks

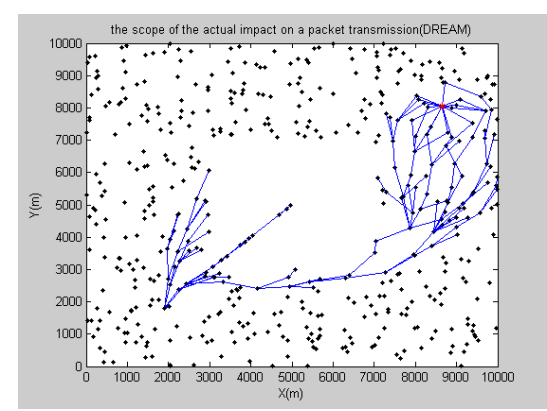

Figure 2. The actual route given by DREAM 

on Underwater Acoustic Networks

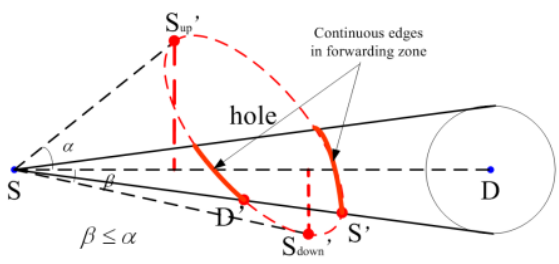

(1) Here we chose $\mathrm{S}_{\text {down }}$ ' as $\mathrm{S}_{\mathrm{d}}$

(2) $\mathrm{S}^{\rightarrow} \mathrm{D}^{\prime} \rightarrow \mathrm{S}_{\mathrm{d}} \rightarrow \mathrm{S}^{\prime}$

Figure 3. The schematic diagram of hole projection

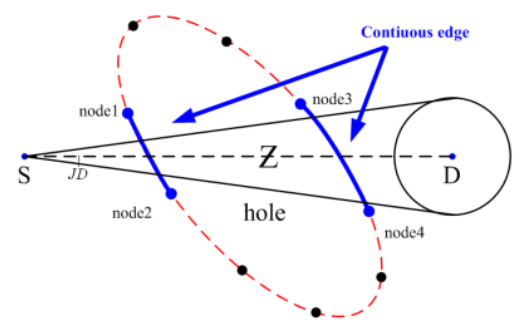

Figure 4. The situation of $K \cap Z=\phi$

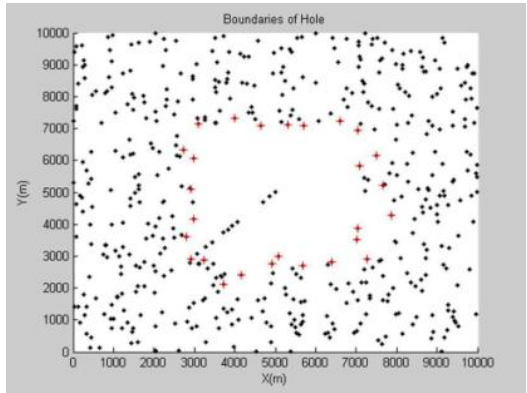

Figure 5. The boundary's nodes detected with the method in [8].

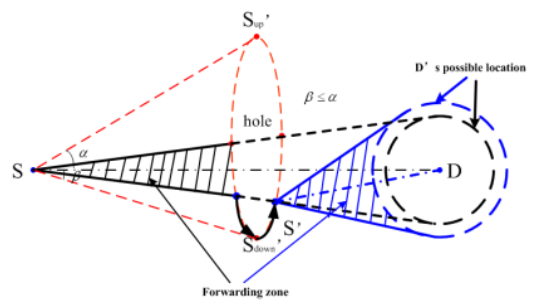

Figure 6. The schematic diagram of packet transmission part 


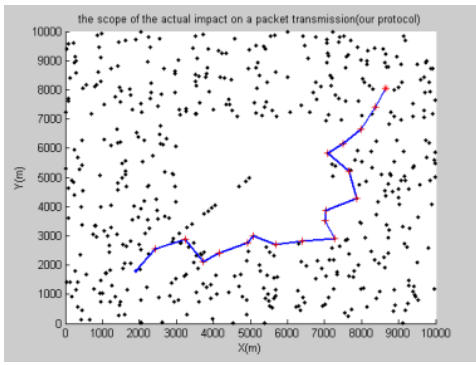

Figure 7. The actual route given by our protocol

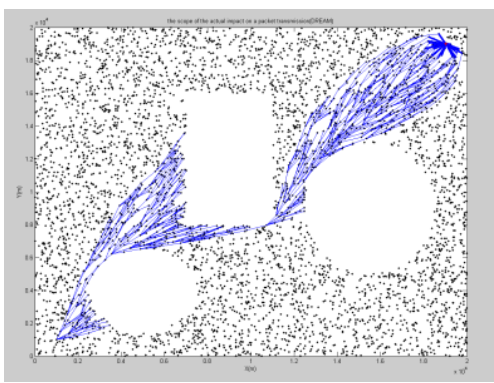

Figure 8. The actual loca during a packet transmission with DREAM

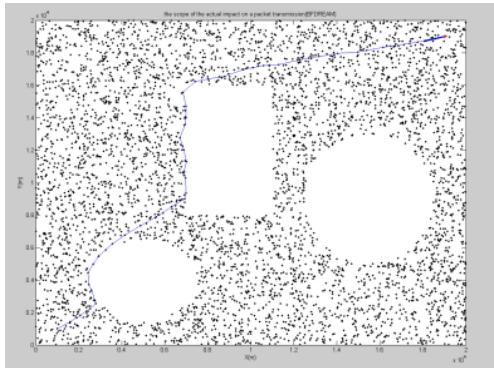

Figure 9. The actual loca during a packet transmission with BFDREAM

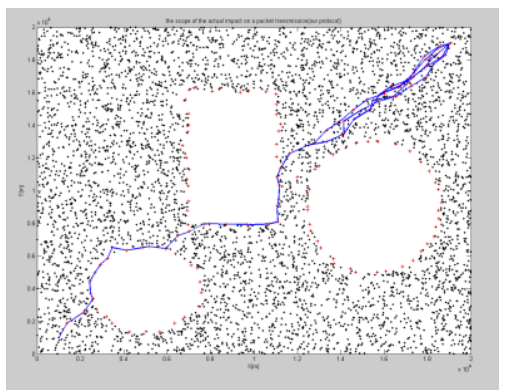

Figure 10. The actual loca during a packet transmission with our protocol 

on Underwater Acoustic Networks

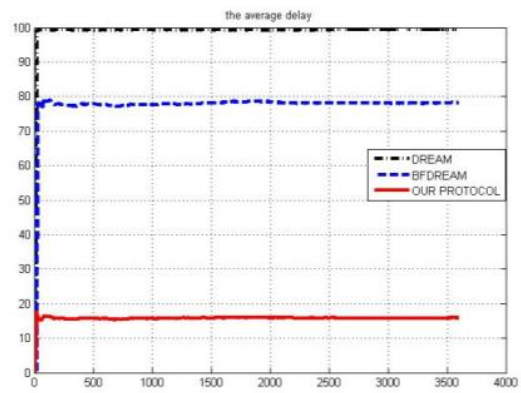

Figure 11. The average delay of packet transmission

\section{NETWORK-ENERGY}
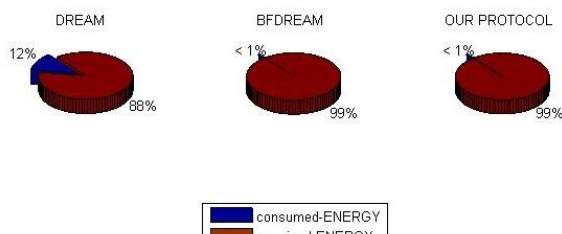

Figure 12. Energy Consumption

\section{NON-EFFECT-RATE}
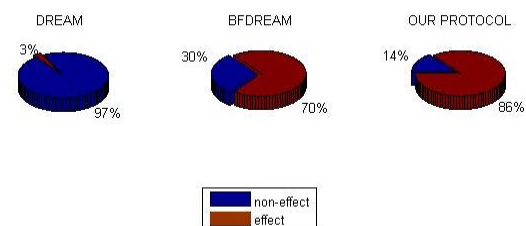

Figure 13. The Ratio between the total load of Non-Effect information and the total load of Effect information 